\title{
The Pattern of the Global Map of Science: A Matter of Contingency?
}

\author{
Cédric Gaucherel \\ UMR AMAP, Écologie des forêts, prairies et milieux aquatiques, Montpellier, France \\ Email: gaucherel@cirad.fr
}

How to cite this paper: Gaucherel, C. (2019). The Pattern of the Global Map of Science: A Matter of Contingency? Open Journal of Philosophy, 9, 82-103. https://doi.org/10.4236/ojpp.2019.92008

Received: October 27, 2018

Accepted: May 5, 2019

Published: May 8, 2019

Copyright (C) 2019 by author(s) and Scientific Research Publishing Inc. This work is licensed under the Creative Commons Attribution-NonCommercial International License (CC BY-NC 4.0). http://creativecommons.org/licenses/by-nc/4.0/ (c) (7) \& Open Access

\begin{abstract}
A consensual pattern in the form of a circular map today emerges from connections between the major (high level) disciplines of science. Classifying sciences is useful in practice, but the resulting pattern in itself is rarely questioned. Here, I suggest that this pattern may, at least partly, be explained by the degree (intensity) of contingency of the objects they study. The contingency property appears to have two distinct yet interlinked origins: the major contingent events that have chronologically built our universe and the minor contingent events building every new object or phenomenon. This study provides a simple mathematical model to formalize causes generating the pattern of the global map of science. I propose to use the $\mu$ parameter of a branching process to more objectively quantify the degree of contingency of each science. Although biased by occidental culture and other confounding factors, the global pattern of sciences certainly provides crucial information on the deep nature of science.
\end{abstract}

\section{Keywords}

Science Hierarchy, Scientific Law, Branching Process, Mathematics, Thought Experiment

\section{Introduction}

How do sciences differ? Sciences may differ due 1) to the differences of the objects they study as well as, due 2) to the way they are studied. But how precisely? Recently, several powerful tools to map science may help answer to this long lasting question (Börner et al., 2012; Klavans \& Boyack, 2009). What are called global maps of science today allows mapping career trajectories, identifying emerging research fields or building profiles of institutions. Such maps use automatic and objective algorithms to compute dominant connexions between 
sciences (i.e. major or high level scientific disciplines) from large sets of sources (Börner et al., 2012; De Nooy, Mrvar, \& Batagelj, 2011). Beyond practical needs and uses, such global maps of science exhibit patterns which would be interesting to interpret (to understand), although this question is rarely listed among the motivations for mapping science (Börner et al., 2012). The present paper aims at revisiting this age-old question on the basis of a new concept, the degree of contingency of a science.

Recent attempts to map science interestingly converged to a consensual pattern, linearly connecting major sciences (Börner et al., 2012; De Nooy et al., 2011). The 1D map may be viewed as the dominant pattern today connecting major sciences between them, and corresponds to the projection of all sciences and sub-sciences relationships into a Riemannian space. To assume a Riemannian space deliberately avoids preferential directions and Cartesian boundaries (top, bottom, left and right) and so, avoids favouring/disfavouring a specific science among others. The last versions of these maps are computed on a continuous sphere then projected into a 2D Mercator map and a summarized 1D map (Figure 1). Interestingly, the locations of major sciences on this consensual 1D map (hereafter called the "map of science", MoS) fit well with the age-old classification proposed to organize them (Comte, 1865; Nagel, 1961). Why does human intuition fit with the dominant pattern of sciences when objectively computed? Does it reflect simple interactions between human ideas or does it hide deeper foundations of the objects of science?

The MoS is a convenient tool providing a common cognitive framework

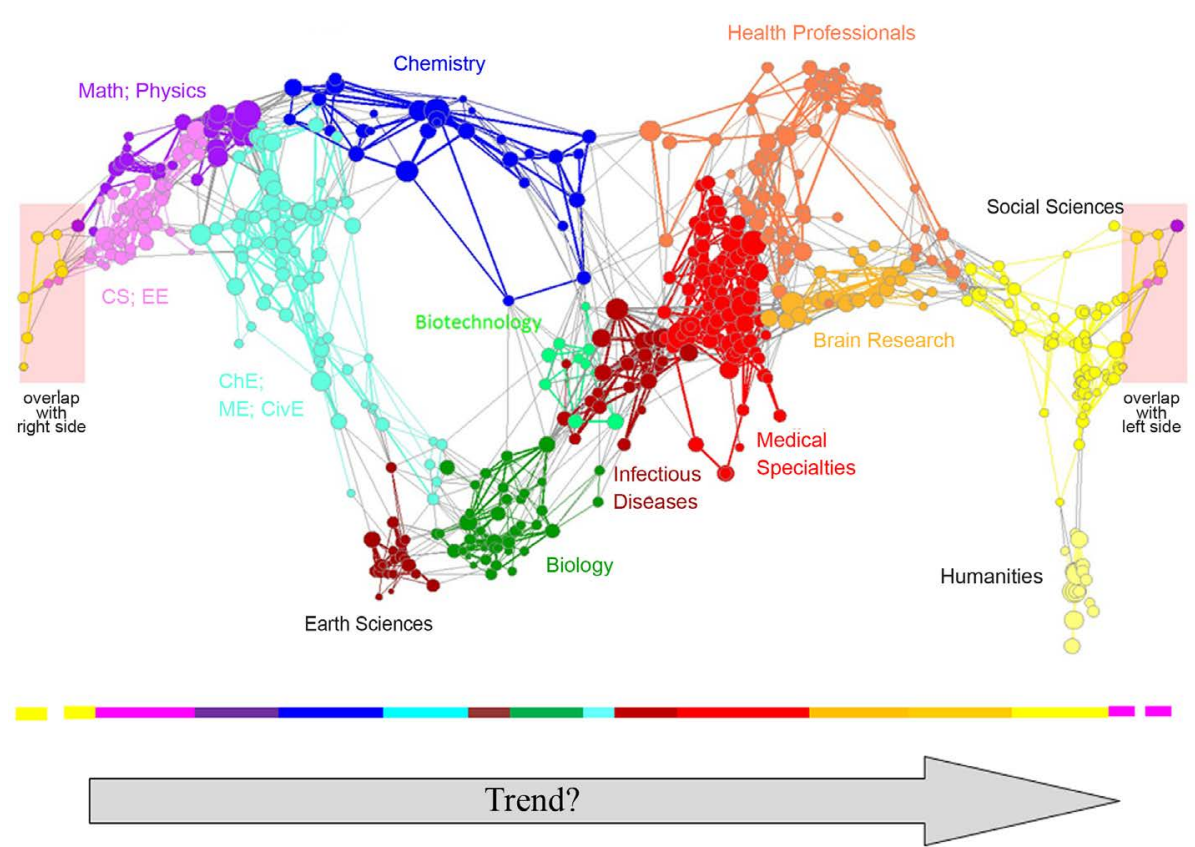

Figure 1. A Mercator projection of the UCSD map of science (top), superimposed to a one-dimensional projection of this map (top). We question here whether the apparent trend exists, although it does not presuppose any inertia (as degree of contingency could occasionally decrease). With the kind permission of authors (Börner et al., 2012). 
available for guiding science policy and education purposes. Simultaneously, the MoS provides a "semantic space" to understand relationships between sciences. Indeed, while specific studies in information science may analyse the science ontologies (Gruber, 1993; Sowa, 1995), they usually focus on one specific domain or object category and rarely compare them at large. Yet, it is relevant to use such a MoS for analysing science relationships. For example, mathematical and physical objects always appear closely linked, whatever the MoS algorithms used, the source analysed and the MoS shapes (e.g. hierarchical, centralized or non-centralized and circular) chosen.

To understand this relationship and others, we question here the relevance of the contingency concept. Among others, contingency has been widely debated in biology, and more intensely since the Gould's famous question: what would happen if we replayed the tape of life? This "evolutionary contingency thesis" expresses the fact that life may or may not follow the same path in similar (initial and consecutive) conditions (Gould, 1989; Haufe, 2015). Although Gould believed to evolutionary laws, he long denied that life would have looked similar in similar conditions. Other scholars answered that frequent convergences in life, in particular when subject to physical constraints, would lead to roughly similar species (Beatty, 1993; Morris, 2010). The debate becomes acute when concerning intelligent species. In physics or mathematics, few of us would venture to question the contingency of their studied objects; some well-defined probabilities settle the stillborn debate. Yet, the question might be relevant for differentiating sciences.

The idea to compare sciences, first emerging from a wish to identify their specificities and a possible hierarchy, culminated with the work of Comte (1865). Later, the comparison was motivated by the search of a possible boundary between "pure" (or hard) and less pure (soft) sciences (Nagel, 1961; Popper, 1963; Putnam, 1975). Now, does such a boundary exist? More generally, is any organization of sciences possible? The MoS recently displayed a consensual organization, thus feeding this long lasting debate. Yet, it remains an open question to understand the dominant pattern connecting sciences. In this paper, it is shown that sciences possibly differ by their degrees of contingency and, as a corollary, that this continuum rules out any hierarchy or sharp differences in nature between sciences. One logical goal of this approach is to provide a more objective process to understand science specificities rather than to subjectively rank sciences.

\section{Problem Statement and Precautions}

One intriguing science certainly is mathematics, often viewed as a highly abstract activity, and thus decoupled from the real world that is investigated by other sciences. This point has been widely debated. For example, H. Putnam proposes that, unlike other sciences, mathematics deals with a priori entities, although not absolute (i.e. out of any context) a priori (Putnam, 1975). This au- 
thor develops the example of non-Euclidian geometries "discovered" during the 19th century, and explains how contingent these discoveries were. Here, contingency should be defined as "something that occurred, but that need not have occurred or could have occurred differently". Here also, contingency should be understood as the quality of being "absolutely contingent", rather than "contingent upon" previous causes (Beatty, 1993). This implies that mathematics is contingent, as a scientific and human activity, but says nothing about mathematical entities themselves. This is why this proposition does not contradict the feeling of most mathematicians met so far: they all see their science as dealing with non-contingent entities (Panza \& Sereni, 2013), whatever the way they work. We may express their view by the thought experiment of someone arriving on another planet and meeting some intelligent life form that should necessarily be aware (according to this non-contingent view of mathematics) of numbers, of prime numbers, etc.

Nevertheless, Putnam's work opened a promising avenue to consider all sciences as part of a continuum admitting some degrees of a priori (Putnam, 1975). This paper proposes that sciences are linked by a continuum of a different kind, made by gradual degrees of contingency of their studied objects. Contingency has been analysed in depth in various sciences (Beatty, 1993; Huneman, 2010), separately, but no study, to my knowledge, uses this property to compare (i.e. to discriminate) sciences. For example, social and human sciences are highly influenced by the contingency of their objects of study; it is now a matter of fact that history (Sterelny, 2015), sociology and some branches of philosophy concern contingent entities (Deleuze \& Guattari, 1991; Stengers, 1995). This debate is even warmer inbiology and living sciences, for the reason that we know (so far) a unique sample of life and that we can hardly imagine something else (Gould, 1989; Morris, 2010; Pearce, 2012). Yet, this paper will not discuss contingency in biology per se, still highly debated elsewhere (Haufe, 2015; Mitchell, 2000).

The focus here is on objects of science rather than on the human activity of science. Life (the studied object) and biology (the science) differs. Nobody will deny that biology, as other sciences, is a contingent human activity. Conversely, we still ignore whether living entities, and some other objects studied by sciences, are contingent. In addition, while sciences are human constructions, I assume here that each science studies real objects uniform enough (in terms of properties), and showing boundaries sharp enough to be unequivocally differentiated from those of other sciences. Even if such an assumption reveals irrelevant, it does not prevent trying grasping some general properties on sciences. here, we consider a science defined as a branch of knowledge subject to a scientific approach (Carnap, 1974; Gaucherel, 2012), and consider sciences as very coarse-grain and relatively homogeneous constructions. This definition fits well with the major (often 16 high level) disciplines of science proposed to simplify maps of science into the consensual MoS (Klavans \& Boyack, 2009). Yet, it is out 
of the scope of this study to discuss the way sciences appear and interact, rather than to compare them on a more objective basis.

An in-depth examination of the contingency of various sciences would demand prior knowledge of their specificities, as well as of the differences between them, or, more precisely (Iinsist), between the entities they cover. Whatever our point of view, the differences between sciences should not be impacted by the way we see the world, such as from a realist (or the opposite) standpoint, because we have no reason to suppose that some sciences would require real objects (of a specific type) and others may not. Differences in contingency between sciences may be illustrated by the role of past contingencies in the construction of our world and the role of (supposedly different) everyday contingencies in each science (mainly mathematics, philosophy, physics, sociology, biology, psychology, and chemistry). This chronology and the successive events within each science are partly responsible for the contingency found in scientific knowledge. Thereby, I will cover the intuitive classification of almost all sciences (Comte, 1865; Nagel, 1961), while staying clear of any hierarchy. Finally, I will then examine the possible reasons for the variations we observe in their contingency, as well as their consequences, particularly in terms of the scientific activities in each science.

\section{Our Contingent World}

\subsection{Contingent or Non-Contingent?}

Our world is contingent. The world that surrounds us depends on the happening of some striking events (Gell-Mann, 1994; Maynard Smith \& Szathmáry, 1995) and, consequently, it is probable that different striking events would have radically changed the world's fate. However, we are not sure whether other striking events could have occurred. For example, we today observe a unique sample of the universe (ours) and a unique sample of life (on Earth), and we do not know whether other universes and/or forms of life exist. We have therefore been unable, so far, to conclude that both universe and life are contingent (Mayr, 2004). Conversely, both our common sense and our current theories (which include stochasticity) tell us that our universe and life might have been different, thus pointing to the possibility that these events and objects are contingent. For the sake of demonstration, I will assume that the major (striking) events at the origin of the universe are contingent, and I will also discuss this assumption further. Yet, this assumption will have a weak impact on our conclusion, given that the debate here focuses on "differences" between sciences, rather than on the intrinsic (absolute, and possibly unreachable) contingency of each science.

The most abstract objects that science handles are possibly mathematical entities, arbitrarily set at the top of the consensual MoS (Börner et al., 2012). The movie Avatar (directed by James Cameron) was interesting for a reason rarely mentioned: it asks how contingent our world is. Following the thought experiment of mathematicians arriving on Planet Pandora, would you believe that the 
Na'vi people had knowledge of numbers and geometry? Almost all mathematician colleagues answer in the affirmative, although this long-lasting debate in philosophy of mathematics between Platonism (i.e. mathematical objects pre-exist) and nominalism (there is no abstract objects) is still open (Panza \& Sereni, 2013 and references therein). Very few mathematicians recently suggested another point of view (Nelson, 2001). This debate is beyond the scope of this paper. Would H. Putnam have answered negatively, arguing that we could find at least one planet on which an intelligent life form would not necessarily know numbers and other mathematical entities? He has in fact put forward such an argument using examples in geometry and quantum mechanics of contingent mathematical discoveries (Putnam, 1975). However, Putnam apparently does not distinguish between mathematical entities and the history of mathematics. It is one thing to say that mathematics is contingent, as it is a human activity, and another one to say that mathematical entities are contingent.

Next, how can we be sure that human intelligence and human logic are unique? If we were to find another intelligent form of life, building its environment on the basis of a different logic from ours, this would unequivocally demonstrate the contingent nature of the mathematical entities that we know. Three studies provide some (admittedly weak) clues that a different type of reasoning than ours is possible. Surprisingly, the first clue comes from the biography of the greatest logician of the past century, K. Gödel, and showing during his "elusive deliriums" that coherent logics of another kind could emerge (Cassou-Noguès, 2007). This also fits with the emerging feeling of some mathematicians that contemporary mathematics remain fragile (Nelson, 2001). The second clue may be found in computing science literature, today trying to imagine different kinds of "intelligence" than ours to conceive artificial life. One may have to look for the third clue in studies of our brain during dreams (Oudiette et al., 2012). The authors of the last-named reference report their discovery that a brain in which prefrontal, parietal, and posterior cingulate cortical parts are missing is unable to construct a coherent story. This finding may point to the possibility of an alternative but still coherent view of the world. As a human being, we are unable to define what a "different logic" than ours may be. Consequently, we cannot be certain that our logic is the only possible logic with which to construct a coherent and viable world, and thus, that our mathematical constructs are contingent.

Intuitively, our physical world is more contingent than are abstract mathematical entities. Physics is the next major science located right to mathematics in the consensual MoS (Börner et al., 2012). It is a matter of fact that our world is associated to several universal constants, such as the Planck constant and the speed of light, whose origins are still obscure (Gell-Mann, 1994; Klein \& Spiro, 1996). We are still unaware if they are related to any specific event that occurred in the past. Rather, we may focus on the history of the universe as physics today describes it. Starting from the primitive "hot soup" of particles, a progressive expansion and cooling of the universe occurred. This cooling consequently separated the four physical forces initially unified, first gravitation with the other 
forces (estimated at around 1019 Giga electron Volts), then quantum chromodynamics separated with the still unified electromagnetic and weak interactions (at $1015-1016 \mathrm{GeV}$ ), and finally the two latter forces (separated at $103-104$ $\mathrm{GeV}$ ). This global cooling is responsible for what could be considered as the early "first contingency" of the physical world (and its components). We still do not know whether the cooling of the universe or the timing of this cooling could have been different (e.g. it might have been delayed), but we do believe that both occurred.

A detailed study of these physical forces reveals their symmetric nature, that is, their behaviour remains unchanged when their signs are changed in equations. The variables concerned are charge $(\mathrm{C})$, parity $(\mathrm{P})$ of particles, and time (T) (Gell-Mann, 1994). In quantum mechanics, the CPT symmetry is conserved, by which we mean that simultaneous changes of sign of these three variables do not result in equations that conflict with empirical derivations, and which are therefore compatible with the one specific fate, and not another (assuming that they could have been different). Consequently, contingent events belong to one of two distinct categories. A contingent event may either be a striking symmetrical event, or a minor event. We may call the former a major event, meaning thereby that it may have occurred once, and have endured up to the present observation time. All major events occurred in a specific way, but they could have occurred differently. In particular, our microscopic world is intrinsically stochastic, and physical laws of quantum dynamics are correct statistically only. In most cases, we observe an undetermined product of particle interactions, such as the $\mathrm{K}$ meson disintegrating real world in which particles with opposite charge and parity interact in reversed time. We have known since the pioneering experiment of 1964 on neutral $\mathrm{K}$ meson that the CP symmetry is broken (Christenson, Cronin, Fitch, \& Turlay, 1964). Considering that CPT symmetry is always conserved while $\mathrm{CP}$ symmetry may not, it seems reasonable to conclude that the $\mathrm{CP}$ symmetry breaking is responsible for time irreversibility (i.e. for $\mathrm{T}$ symmetry breaking, because $\mathrm{CP}$ and $\mathrm{T}$ changes impact the $\mathrm{CPT}$ symmetry, (Klein \& Spiro, 1996). A. Sakharov even proposed that the CP symmetry breaking might be responsible for the lack of antimatter in our universe, although this today remains a hypothesis. So, we deduce from these observations that the quantum universe, mainly composed of matter, has a time arrow. Hence, the time symmetry breaking resulting from the global cooling appears possibly the first major and the most striking contingent event of the universe.

All symmetry breakings may be considered contingent events, as they lead to sometimes in two, sometimes in three $\pi$ Mesons (Christenson et al., 1964). Particles themselves can be described as waves and fields and may be present in several states simultaneously (Gell-Mann, 1994). These observations lead to the inference that physical objects, despite their laws, are partly contingent. By the way, this observation is valid whatever the physical object, thus giving a uniform view of this science regarding to these properties.

This observation on contingent objects in physics is also true for thermody- 
namics and chaos theory, the physics of the macroscopic world, whose second principle states that all reactions occur in one direction (the direction of increasing entropy) and not in the opposite direction (Brillouin, 1956; Carnot, 1824). It took a long time to understand the origin of the second principle, but we now confidently interpret it as the consequence of the large number of entities composing an object (Balian, 1991; Klein \& Spiro, 1996). This large number indeed prevents a return to the previous state of the system, with a transition probability much lower than in the case of a low number of entities, thus imposing a direction in changes. This irreversibility corresponds to the orientation of time, and thus to the first of the steps that would progressively build the history of the system. Here again, we observe a symmetry breaking which, every time it occurs, produces a contingent object. This time asymmetry thus explains how we can observe this particular planet which is Earth, with its specific history, its specific structure, with its plate tectonics. As a corollary, geology is, beyond its object diversity, a contingent science too.

\subsection{Non-Physical Sciences}

Following the cooling of the universe, atoms combined into molecules of increasing sizes and complexity. Chemistry, the science of these objects, is the next major science in the MoS (Börner et al., 2012). Later on, an astonishing instance of contingency is to be found in the chirality of proteins (i.e. one which cannot be superimposed on its image in a mirror) and of many molecules. Although, in theory, nothing preventing us from observing sinistral (left-handed) proteins, the chemistry encounter in life observes dextral (right-handed) molecules only. For example, this is the case with the myoglobin composed of 153 amino acids, a strongly chiral molecule. This observation provides another instance of symmetry breaking, here in space, as it does not allow a certain kind of spatial orientations for all molecules that will then interact with the early sinistral molecules (Balian, 1991). This other event, also still unexplained, appears to be, beyond the molecule diversity, another major contingency.

Going beyond the atomic relationships, should we not wonder whether the Mendeleev classification of elements itself is contingent? Else why these elements? Why this organization of matter? Why these specific properties? Similarly to the universal constants, the Mendeleev classification could not clearly be associated with a specific event. So, we could not consider it as a major contingency. This is why I focused here on a chemical event (the appearance of chirality) that could have occurred differently (e.g. sinistral) on another planet. In brief, if chemistry is the science of combining simple elements into sometimes more complicated ones, the objects of this science are therefore contingent as some associations could occur and some not (Bersini \& Reisse, 2007; Saitta \& Saija, 2014). As another illustration, our planet every day uses petrochemistry that is today well developed, while it is predictable that some other planet would not experience (or even know of) this context. 
Chemical entities had once constituted a living entity, one of those that biology today studies, the next major science in the MoS (mixed with engineering and earth sciences). Those engaged in that pursuit are faced with a challenging task: identifying what in living entities is contingent and what is not. The life we know is built on the carbon element and on the genetic code (e.g. Mayr, 2004). Could it have been different? We still do not know, although we have the feeling that other forms of life could be based on silicon (a chemical analogue of carbon) and on another code (Bersini \& Reisse, 2007; Gaucherel \& Jensen, 2012). For example, genetic code uses the four A, T, G, C codons and 21 amino acids, but nothing prevents life, in theory, from using more codons, other molecules as codons and other amino acids or protein types. We know that these molecules have been selected for their efficiency in maintaining life, but why should we not imagine that other combinations would be similarly efficient? Why should we not imagine, even, that other combinations of atoms would be as (or more) efficient if prebiotic entities had taken another trajectory, early at the beginning (Fontana \& Buss, 1994; Gaucherel \& Jensen, 2012; Saitta \& Saija, 2014)? This hypothesis does not necessarily challenge natural selection or other biological principles or laws.

These primitive stones building life being fixed, life can develop in a way that we start understanding today. It has been a recurrent question since Stephen Jay Gould's work to examine whether the history of life, when "replayed", would follow a similar trajectory (Gould, 1989). Seven and sometimes eight major stages have been identified in this history of life (Jablonka \& Lamb, 2005; Maynard Smith \& Szathmáry, 1995). This is, in essence, what is suggested on Pandora in the Avatar movie, on which we observe other (intelligent) life and other ecosystems populating that planet. Here again, the debate is still open: on the one hand, it has been shown that physical constraints would certainly lead to biological convergences and to similar organisms (Fontana \& Buss, 1994; Morris, 2010), while, on the other hand, life seems to be highly dependent on past catastrophes and past (major) events. Hence, we cannot be sure life is the result of chance only (Beatty, 1993; Vermeij, 2006).

This question of biological contingency is still a hot topic, and it is out of the scope of this essay to discuss the contingency issue in biology (Haufe, 2015; Mitchell, 2000). Yet, biological contingencies, at local and probably global scales, are much more contingent than physical and chemical contingencies (Beatty, 1993; Huneman, 2010). Indeed, the fact that living organisms are based on a more complicated architecture at a higher level of organization is sufficient to indicate that, statistically, they depend on a higher number of past events and contingencies. Life needs the contingent genetic code, which needs contingent chiral molecules, which need contingent matter (and not antimatter). No doubt that biology studies historical entities that are contingent in one way or another (Gould, 1989; Mayr, 2004; Pearce, 2012). Humans are mammals, which are animals, which are sexual species, which are eukaryotes, etc. The transition to each 
new step is at least partly contingent, and will continue to be. It would be relevant to build a contingency indicator to quantify such differences, a point that will be discussed further on.

\subsection{Human Sciences}

Continuing this story of Earth, not only is life contingent, but each individual, each of us is too. A wide body of studies in health sciences and related works then appear in the MoS (Börner et al., 2012). An obvious and critical difference between particles and individuals, such as mammals, is that the former are all rigorously identical, as far as we know, while the latter are all rigorously distinct. This is mainly due to the number of elements that form individuals and to the history of these elements and of the assemblage (Pesic, 2002). Each of us is the result of the evolution first, of our parents' (ancestors') legacy and of our (environmental) influences then. This is why human twins may appear different, although they have a highly similar genome. They have a trajectory that leads each of them to a specific behaviour that biology and psychology study in depth. Psychology and other cognitive sciences study highly contingent objects (Davidson, 1970; Nagel, 1961). Furthermore, these objects are "more" contingent than the objects in biology, chemistry and physics, because they depend on more events (i.e. on both the earlier major contingencies already described and on additional minor contingencies). And they will continue to be so.

Finally, this story continues by observing that human sciences such as history and sociology (i.e. their objects) are contingent too. Human and social sciences then follow on the consensual MoS. First, they depend on the contingent appearance of humans. Then, they depend on the appearance of organized civilizations which occurred in some places on Earth, under certain circumstances, and not others. We are the inheritors of early societies built in Africa, in the Fertile Crescent and in Asia, the early organizations of human beings (e.g. Diamond, 1997). Despite some constants in human organization (e.g. writings and myths, D'Huy, 2013), it is highly probable that several properties of societies remain highly contingent. Natural influences such as climate catastrophes and human influences such as wars are good examples of hazards occurring in the growth of a civilization (Diamond, 1997). This is why history often questions its own heuristicity, in the discussion between D. Hume and N. Condorcet (Condorcet, 1794-1795): "Esquisse d'un tableau historique des progrès de l'esprit humain", as its objects of study are contingent and possibly do not follow any invariant principle (Condorcet, 1794-1795; Deleuze \& Guattari, 1991). We can assert that societies and cultures would be highly different on other continents, at other epochs, and ... on other planets.

It may be worthwhile concluding this survey with a reference to the philosophy of sciences that has been perceived as a contingent activity (Deleuze \& Guattari, 1991). In particular, epistemology is highly contingent, as already mentioned for the philosophy of mathematics (Stengers, 1995). Yet, as a large field of 
thoughts (i.e. a coarse-grain analysis), philosophical themes do not possess a uniform degree of contingency, whether they concern logic, ethics or metaphysics. Even if the entities studied by a particular science are non-contingent, it is probable that their "discovery" by an intelligent life would be highly contingent, as demonstrated by the trajectory of geometry (Putnam, 1975). This is more obvious for clearly contingent sciences for which the study interacts with the studied object (e.g. social and economic sciences), and to consequently modify this object. The discovery of non-Euclidian geometries then encouraged scientists to look for such geometries in Nature. We can predict continuous interactions between the object and its study, such that neither would follow a predetermined trajectory. So it is crucial to study such objects on the basis of logical propositions, as this allows for separating the contingency of philosophical objects from the (invariant) non-contingency of the hidden principles they obey (Barberousse, Kistler, \& Ludwig, 2000; Carnap, 1974). This is also possibly what is occurring in quantum mechanics, as the state of each microscopic system is never independent of the observation, and of the observer (Gell-Mann, 1994; Putnam, 1975).

Interestingly, the circular MoS then connect human sciences to mathematics through computer sciences and related fields. This observation is a direct consequence to the choice of a Riemannian geometry. For worthy reasons and to not favour any science, this geometry assumes that no border should limit science maps. Yet, in case we assume to prune the circular MoS, the most intuitive aperture among the 16 high level sciences usually listed is probably located between mathematics and human sciences (Börner et al., 2012; Gaucherel, 2013; Putnam, 1975). We remind that it is out of the scope of this study to discuss the way sciences appear and interact, rather than to compare them on an objective basis. Yet, the weakest link in the consensual MoS is to be found between mathematics and computer sciences (Figure 1) (Börner et al., 2012; Klavans \& Boyack, 2009). I propose here that this observation is not fortuitous or explained by sciences themselves, but due to the contingency of the concerned objects. Between non-contingent mathematical objects and highly contingent social objects, are mapped computer sciences benefiting from the mathematical rigour and abstraction, and often applied to contingent and historical objects (e.g. societies, individuals or psychologies) (e.g. Dean, Kendal, Schapiro, Thierry, \& Laland, 2012; Gaucherel, Boudon, Houet, Castets, \& Godin, 2012). This observation also reinforces the hypothesis that computer tools provide potentially fruitful approaches to understand more contingent objects.

\section{Causes and Consequences of Contingency}

I propose a simple methodology to gain objectivity in this philosophical question. In a first step ( $\& 4.1$ ), I review differences between contingencies of various sciences and propose a contingency index to characterize each of them. In a second step, I develop a simple model to help quantifying, at least qualitatively, 
this index in most sciences involved in the MoS (\& 4.2). For this purpose, a branching process will be used to grasp the way events occur in each science studied. In a third and last step, I apply this model to the main sciences to better fit it to our common sense and to $\operatorname{MoS}(\& 4.3)$. Results are then discussed in an epistemological perspective ( $\& 4.4$ ).

\subsection{A Degree of Contingency}

All these striking events thus cumulate in time to create the world we know, the world we live in, the world we study. The list above (nine major events) is not exhaustive, but even without additional knowledge, we can bet that the creation of time, a matter-dominated universe, chiral molecules, the Earth and its continents, a carbon-based life with a specific genetic code, the human species, societies and the greatest scientists (as individuals) are all contingent. Let's change one of these major events, and the world would be radically different, for sure. Due to this chronology, objects of a certain science or discipline appearing subsequently lead to a more contingent science, as this science depends on a higher number of past major and minor contingencies.

More importantly indeed, each science today continues to depend on ongoing (minor) contingencies. Due to the nature of these objects, be they stars, continents, animals or societies, they inherit the properties and the contingency of their categories (i.e. of their coarse-grain sciences). For example, all mathematical entities probably have the same abstract nature and the same lack of contingency, the same a priori nature according to Putnam. He suggests that other sciences have "less a priori" (Putnam, 1975). We understand it as an attempt to point to a variable's contingency. Physics studies entities that are slightly more contingent than mathematical ones, not only due to the primary (major) contingency found in the matter-antimatter disequilibrium, but also due to everyday (minor) physical interactions. The undetermined productions of innumerable particle interactions, the stochastic variations of thermodynamical systems, the fate of our chaotic planetary system (chaos determinism may nevertheless be highly contingent), the spatial arrangement of the continents-all these objects are contingent and will continue to be, every day (Gell-Mann, 1994; Klein \& Spiro, 1996).

I suggest calling these instances "minor" contingent events, in the sense that they continuously occur today. The probability distributions of these minor events could differ from those of the nine major events previously mentioned (Gaucherel, 2013; Gell-Mann, 1994; Gould, 1989; Jablonka \& Lamb, 2005; Maynard Smith \& Szathmáry, 1995). Yet, without additional information, I will here assume (as a working hypothesis) that they are of a similar type. Furthermore, nothing prevent us to hypothesize that the major events initially were minor events that froze one day in the past, with much higher consequences than the other (non-frozen) minor events. Breaking symmetries, chirality and life once became major events in their related sciences, because they were the first ones or 
for some other unknown reason.

Similarly, a huge number of objects, appearing later in this chronology of the universe, are built on higher levels of organization. Therefore, they are statistically dependent on a higher number of contingencies and they continue to depend on additional contingencies. Molecules we find on Earth's surface are chiral, have a certain range of temperature activation and are often based on carbon (Maynard Smith \& Szathmáry, 1995; Mayr, 2004). However, the products of their reactions are often contingent, although they follow chemical laws (Saitta \& Saija, 2014). Similarly, at a higher level of organization, life depends on this chemical contingency in addition to the intrinsic contingency of living entities. Even if life continues in future to be based on the genetic code we know, the diversity we observe in species and individuals will continue to evolve. This evolution is, at least locally, highly contingent. It depends on contingent mutations and contingent environments. Here, our aim is only to highlight that life is more contingent than inert objects, and we will later propose a way to quantify this difference. At least two reasons, in deep interaction, may explain why: 1) life comes later in the universe's chronology, and 2) life involves a higher level of organization and so shows higher chances to show bifurcations of events (Balian, 1991; Bersini \& Reisse, 2007).

This observation is even more relevant for human sciences, as they are based on life and presuppose higher levels of organization between living organisms. Due to the history of life and of human beings, the objects of these sciences, social, linguistic and economic sciences, appear highly contingent. A debate concerning an ontological reduction of sciences (Fodor, 1983; Nagel, 1961) is beyond the scope of this paper. But philosophy provides a relevant example of multi-realization in human sciences. For example, I. Stengers adopted an externalist posture by analysing science as the result of the objective works of scientists and their political and sociological beliefs (Stengers, 1995). She proposes science as the trade-off between the contingency of the world and the non(less-?) contingent constraints of scientific activity. Yet, the contingency in philosophy is less easy to estimate for topics in logic or metaphysics which, with their links to mathematics and universal questions, possibly involve non-contingent considerations. As in most sciences and as already said, we should warn about the dangers to average contingencies of contrasted objects in the same science. We may certainly identify some coarse-grain classification of sciences gathering relatively homogeneous (similar) objects, and allowing discussing their common properties (e.g. contingency).

\subsection{A Contingency Metric}

A metric of contingency that is not subjective would be valuable in our discussion. Although such quantification is probably an unattainable dream, we can, at least theoretically, define it on the basis of a "branching process". Branching process theory has been proposed in probability theory to rigorously study 
population growth based on the reproduction of its individuals (e.g. Grimmett \& Stirzaker, 1992; Saunders \& Wallace, 2008). Each individual in generation $n$ produces some random number of individuals in generation $n+1$, according to a fixed probability distribution that does not vary from individual to individual. It is straightforward to define event succession in any science as a branching process modelling the way any event leads to no event, to one event or to several new events at the next $(n+1)^{\text {th }}$ observed time step:

$$
Z_{n+1}=\sum_{i=1}^{Z_{n}} X_{n, i}
$$

In this recurrence equation, $Z_{n+1}$ denotes the state of the event ensemble at time step $n+1$, which may be here interpreted as the number (the ensemble size) of possible events at generation $n+1 . X_{n, i}$ is a random variable denoting the number of direct successor events of event $i$ at time step $n$. It is worth the trouble to list the hidden (and probably approximate) assumptions made here, as we have no choice but to admit at the outset that events should be normally distributed, that this probability distribution should be fixed, and that the $X_{n, i}$ events are independent and identically distributed random variables over all $n \in\{0,1$, $2, \ldots\}$ and $i \in\left\{1, \ldots, Z_{\mathrm{n}}\right\}$. These assumptions linked to the probability distributions of objects will be discussed below.

As in any branching process, the expected number of possible events following each event is defined by $\mu$, the mean of the probability density of the event (normal) distribution:

$$
f\left(X_{n, i} \mid \mu, \sigma=1\right)=\frac{1}{\sqrt{2 \pi}} e^{-\frac{\left(X_{n, i}-\mu\right)^{2}}{2}}
$$

without additional information, I have assumed here a standard deviation $\sigma$ (and variance) equal to unity. The expected number of events generated at time step $n$ is equal to $\mu^{n}$. A central question in the theory of branching processes is the probability of ultimate extinction, where no individual (here, no event) would exist after some finite number of generations:

$$
\lim _{n \rightarrow \infty} \operatorname{Pr}\left(Z_{n}=0\right)
$$

In our case, $\mu$ is the number of new events, a strictly positive integer too ( $\mu \in$ $\{1,2 \ldots\})$. Indeed, the absence of new events in average $(\mu=0)$ is not realistic (or of no interest) in our world. Hence, two different cases remain: If $\mu=1$, then ultimate extinction occurs surely (with probability 1 ) unless each event always generates exactly one new event; alternatively, if $\mu>1$, then the probability of ultimate extinction is less than 1, but not necessarily nil, and potentially high (say, if $\mu>>1)$.

Following this formalism, I suggest $\mu=1$ to describe the average number of new events of a non-contingent science (as mathematics could be), while $\mu>1$ would concern more or less contingent sciences. The "degree of contingency" may precisely be the $\mu$ parameter. The higher $\mu$, the more contingent the associated science. So, an event $i$ is more contingent than another one $j$ as long as their 
respective probability distributions obey $\mu_{i}>\mu_{j}$. Formally, the event $i$ may have been much more different (among $X_{n, i}$ ) than event $j$ (of $Y_{n, j}$ ), because the antecedent event $i$ statistically generates a higher number of events than the antecedent event $j$.

It is obviously not feasible to estimate the contingency, at least with the argument that we are never sure that events which occurred could have occurred differently. A way to proceed to recover (observe) the probability distributions of any object category could be to model typical objects of this science with models able to provide all possible trajectories according to the know processes at play (e.g. discrete systems, Gaucherel, Théro, Puiseux, \& Bonhomme, 2017), and then to quantify the branching process $\mu$ associated. Yet, if only some of these events are contingent, the fact that they are more and more numerous in time (as in the "major event chronology") and that they continue to happen every day (due to the intrinsic nature of the objects) is sufficient for one to qualitatively observe an increase in contingency between sciences and entities. The longer history an object has, the higher its contingency (Figure 2). With a realistic posture in epistemology,

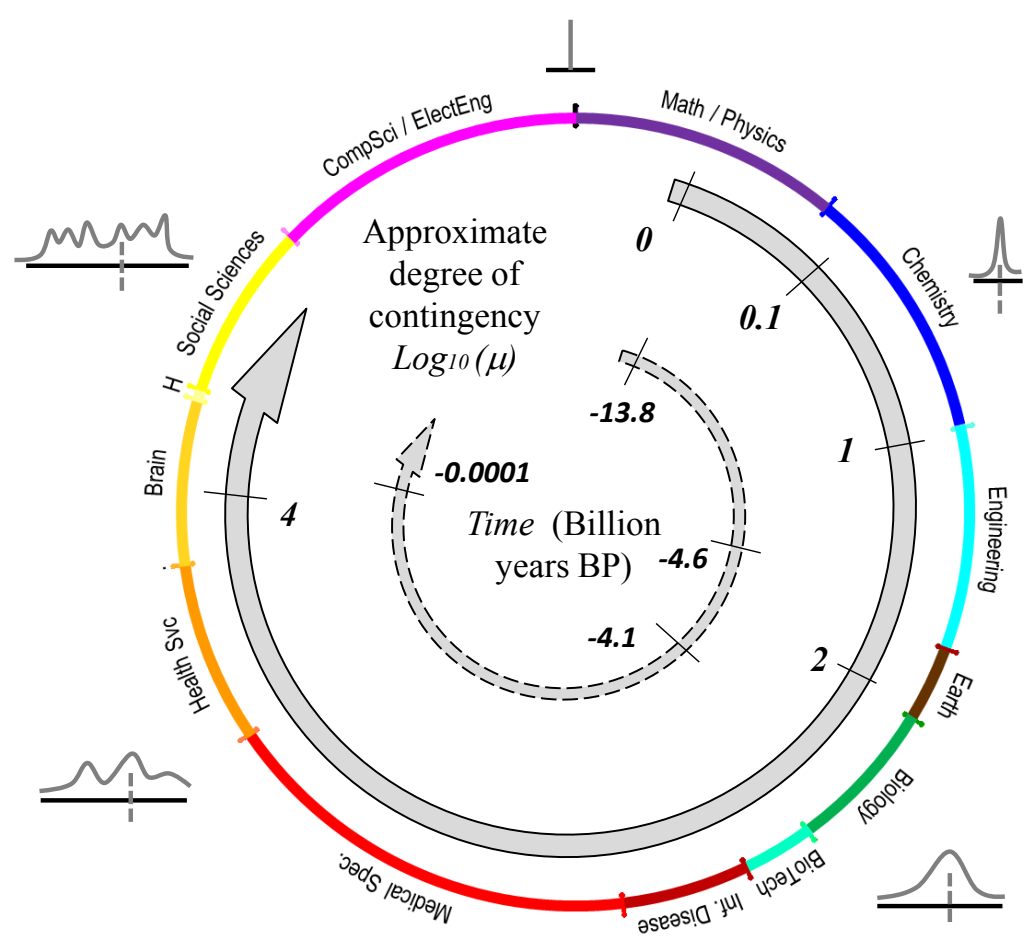

Figure 2. Illustration of the chronological increase of the degree of contingency between sciences. The 2D map of science is projected into a consensual 1D circular map, and is used here as a background for displaying the increase in degree of contingency across time (approximate dates in billion years BP) since the origin of the universe, through the origin of Earth and life, till the appearance of human societies. Typical probability distributions of randomly selected events for each science are displayed (in a $\log _{10}$ scale) for illustration, with their average number of new events $\mu$. The real (observed) probability distributions of each science are unreachable, but may be estimated on the basis of dedicated models exploring all possible trajectories of the same category of objects. With the kind permission of authors (Börner et al., 2012). 
this increase in contingency is real; with an anti-realistic posture (Barberousse et al., 2000; Van Fraassen, 1980), this increase in contingency is a perceived change only, but it still differentiates sciences. Hence, the degree of contingency of a science seems to be a relevant criterion to characterize it.

\subsection{Contingency and Laws}

If this view has some grounds, we may wonder where does come this $\mu$ parameter of the event distribution. In this paper, the central question is not whether a scientific law may have been different, but rather whether, given a law, an event obeying this law may have been different. I do not intend to question the law's contingency (or the contingency of science per se), which has also been a subject of an ongoing debate since the 18th century. Great philosophers (e.g. E. Kant and G. W. Leibniz) discussed whether laws, and in particular laws governing (physical) movement, are contingent or not (Tonelli, 1967). Here, I will consider laws determining the different temporal trajectories that may characterize an object, or an event, and will then comment on the contingency of those trajectories, not on the contingency of the laws themselves. By scrutinizing their respective objects, I here aim to identify a criterion that may have the capacity to help organize sciences as observed in the MoS (Figure 1, Börner et al., 2012).

Recently, it has been proposed to differentiate sciences by the "probabilistic character" of their laws (Gaucherel, 2013), a pragmatic view which is apparently not far from others (Mitchell, 2000). A probabilistic law expresses the fact that an event verifying this law has a certain probability, possibly lower than unity, to occur differently to what the law dictates. This proposition provides a causal way to understand differences between sciences. The contingency discussed here, indeed, is a direct consequence of this probabilistic view of sciences and of the results of their laws: the more "robust" a law (i.e. with a logical probability close to unity), the less contingent the science (Gaucherel, 2013; Van Fraassen, 1980). Indeed, a robust law leaves no other option for the event but to obey the law, thus leading to a statistically lower number of options for every new event.

Now, is the law's probability intrinsic to each science or is it the result of the ignorance we have about its contingent phenomena? We will probably never know, because in our world we usually observe one realization of events only. The next section will explain why contingency may likely be an intrinsic property to each science. Events show one facet only of the objects in a science, and it is difficult, if not impossible, to know what the other realizations of these events would be for a contingent science, except maybe with the proposition to model all possible trajectories of some objects with dedicated models (Gaucherel et al., 2017). These realizations are precisely summarized into the $\mu$ parameter of the event distribution. This conclusion should not prevent us looking for the probabilistic laws and trying to lessen our ignorance of their related phenomena, because these laws precisely gather the non-contingent (invariant) part of the studied entities in a discipline. I admit that this idea remains quite speculative, 
but it may open tracks to understand differences between sciences.

\subsection{Contingency: Discussion and Consequences}

It is useful to have an objective criterion to differentiate various sciences: according to this essay, sciences are more contingent than others when their objects appear later and when they depend on a higher number of contingent events. This observation partly explains why the contingency criterion converges to the usual science ranking (Figure 2). Yet, this ranking is not used here to classify sciences, rather than to improve our understanding of their objects of study. Other factors may potentially play a role in this overall contingency (thus suggesting a need for additional studies), but do "major" and "minor" events play tremendous roles here? Where could such a degree of contingency find its origin? Two reasons may justify the concept of degree of contingency: 1) the history of objects and 2) their organization levels.

It is quite intuitive that a reversible phenomenon is non-contingent, or less contingent than irreversible ones, as it can recover its previous states easily and frequently (but not systematically). Contingency is a direct consequence of irreversibility in time. In other words, irreversibility and contingency are significantly correlated. After several irreversible phenomena have occurred, the contingency becomes almost compulsory, as it is more and more difficult to recover the initial state and to bifurcate towards another history (Balian, 1991). C. Bennett proposes to capture the complexity of an object by using the logical depth (Bennett, 1988; Gaucherel, 2014). This measure is a way to quantify computations needed to build a system, and thereby to capture its organization and its history. In quantum mechanics, de-coherence of particles and of events is at the origin of history of macro-events (Gell-Mann, 1994; Klein \& Spiro, 1996). This is precisely what occurred in the cooling universe, a direction that naturally generates a chronology. This chronology, starting with the first major events listed above, partly explains the contingency of the various entities of our world and their related sciences (Figure 2). In addition, the everyday (minor) contingencies of any events in our world continue to feed the contingent nature of each science or entity.

With such an argument, we would acknowledge the contingency of the objects of a science, but not differentiate sciences from one another. For this purpose, we have to observe the natural (and chronological) organization levels every object is made of. It has been often argued that physical entities combine to give chemical entities, which combine to give living entities, which then combine to give psychological and social entities (Davidson, 1970; Jablonka \& Lamb, 2005; Stengers, 1995). Every complex object such as a society is therefore dependent on the (contingent) individuals it is composed of, on the genetic code it is composed of, on the molecules and atoms it is also composed of. It is a contingent entity, due to its construction process, and due to its history. The neighbouring society also will be highly contingent, and possibly more contingent than the in- 
dividuals it is made up of (because individuals may exist without a society, but not reversely) (Sterelny, 2015).

These differences remind one of the well-known hierarchy of the sciences (Comte, 1865; Popper, 1963), but with two critical differences: 1) this is not a hierarchy (embedding) but a difference only, and 2) this classification is explained by the gradual degree of contingency, not by differences in nature, here defined as the average number of events $\mu$ the science is studying. This view of major sciences fits well with the pattern of science objectively computed in the MoS (Börner et al., 2012; Klavans \& Boyack, 2009), yet with one difference. The consensual (1D circular) map is closed, while the degree of contingency supposes a gradient with opposed directions. As said, this circular shape is a consequence of the chosen Riemannian geometry. By forcing the MoS to avoid boundaries, we assume that no non-continuous structures will emerge (Börner et al., 2012). Hence, I question here the Riemannian nature of science used as an assumption in the mapping procedure: is this continuous nature of science a relevant pattern or an artefact? While it is definitely worthy not to favour any science, I have showed that to use preferential direction (with limits or boundaries, e.g. in time) would not necessary subjectively rank them.

Biology is playing a particular role in this graduation. Indeed, it has been explained why some (physical) sciences are weakly contingent, while some other (human) sciences are highly contingent. Biology and living entities settle in an uncomfortable position between these two extremes. In our opinion, this explains why the contingent nature of biology has continued to be a subject of hot debate (Fontana \& Buss, 1994; Gould, 1989; Morris, 2010; Vermeij, 2006). This debate lies beyond the scope of this paper. In this paper, it is only proposed to compare the contingency found in biology to that in other sciences (Davidson, 1970). And biology likely exhibits an "intermediate" contingency (bottom, Figure 2). An accurate estimation of the degree of contingency of biology will be assessed when we are able to generalize what is life, when we have a validated formalization to describe life as we know it and other closely related systems (Carnap, 1974; Gaucherel et al., 2017).

The presence of a degree of contingency variable between sciences has a surprising consequence in terms of the way sciences are constructed. If we accept the thesis that entities of various sciences have varying degrees of contingency, we must then admit that scientists do not work similarly in facing their objects of study. A scientist working in a non- or weakly-contingent science is likely discovering laws that other scientists may have discovered (or will discover one day). This argument states that waiting long enough will necessarily bring to light the said discoveries, as these discoveries pre-exist independently of the finder (Jablonka \& Lamb, 2005; Tonelli, 1967), a Platonist view of the world. In other words, the scientist in non-contingent sciences is "replaceable". It is therefore surprising that the only way for mathematicians to become irreplaceable (indispensable) is to collaborate with other sciences in applied mathematics. 
And the fact that science is a cumulative activity does not weaken this conclusion.

Conversely, a scientist working in a highly contingent science is likely discovering regularities (e.g. probabilistic laws, Gaucherel, 2013) that depend on their historical context and might not even exist in another (spatial and temporal) context. Let's think about life on another planet, studied by a Pandoran biologist. The discoveries made depend on the context of the object, on the context of the scientist and on the interaction between both. Someone else converging to the same question in these contingent sciences, and sometimes to the same observations, would likely make a different assessment and possibly reach another conclusion (D'Huy, 2013; Deleuze \& Guattari, 1991). In a scientific domain, the scientist using the scientific method probably will, Ihope, converge to the same regularity (invariant) and laws, even if they exhibit different probabilities. Here, the scientist is losing certainty, but is gaining originality (Gaucherel, 2012). In this sense, the scientist in contingent sciences is "irreplaceable", as an artist is. There is no doubt that the artistic production of any scientist is highly contingent.

\section{Conclusion}

Finally, although possibly biased by occidental culture, the global pattern of sciences displayed by the consensual map of science certainly provides crucial information on the deep nature of science and its objects. Here, I have explained why the consensual $1 \mathrm{D}$ circular map of science may highlight a variable degree of contingency between studied objects. In addition, to anchor sciences in a time continuum, I have shown that science may have a different structure than the continuous structure we today assume to avoid ranking them.

\section{Conflicts of Interest}

The author declares no conflicts of interest regarding the publication of this paper.

\section{References}

Balian, R. (1991). From Microphysics to Macrophysics: Methods and Applications of Statistical Physics. Berlin//Heidelberg: Springer-Verlag. https://doi.org/10.1007/978-3-540-45475-5

Barberousse, A., Kistler, M., \& Ludwig, P. (2000). La philosophie des sciences au XXème siècle: Paris: Flammarion.

Beatty, J. (1993). The Evolutionary Contingency Thesis. In G. Wolters, \& J. G. Lennox (Eds.), Concepts, Theories, and Rationality in the Biological Sciences (pp. 45-81). Pittsburgh, PA: University of Pittsburgh.

Bennett, C. H. (1988). Logical Depth and Physical Complexity. In R. Herken (Ed.), The Universal Turing Machine: A Half-Century Survey (pp. 227-257). Oxford: Oxford University Press.

Bersini, H., \& Reisse, J. (2007). Comment définir la vie? Les réponses de la biologie, de 
P intelligence artificielle et de la philosophie des sciences. Paris: Vuibert.

Börner, K., Klavans, R., Patek, M., Zoss, A., Biberstine, J., Light, R., Larivière, V., \& Boyack, K. (2012). Design and Update of a Classification System: The UCSD Map of Science. PLOS ONE, 7, e39464. https://doi.org/10.1371/journal.pone.0039464

Brillouin, L. (1956). Science and Information Theory. New York: Academic Press.

Carnap, R. (1974). An Introduction to the Philosophy of Science. Mineola, NY: Dover Science Book.

Carnot, S. (1824). Réflexions sur la puissance motrice du feu et sur les machines propres à développer cette puissance. Paris: Bachelier.

Cassou-Noguès, P. (2007). Les démons de Gödel. Logique et folie. Paris: Seuil. https://doi.org/10.14375/NP.9782251760407

Christenson, J. H., Cronin, J. W., Fitch, V. L., \& Turlay, R. (1964). Evidence for the $2 \pi$ Decay of the K20 Meson. Physical Review Letters, 13, 138. https://doi.org/10.1103/PhysRevLett.13.138

Comte, A. (1865). A General View of Positivism. London: Cambridge University Press. https://doi.org/10.1017/CBO9780511692888

Condorcet, N. D. (1794-1795). Esquisse d’un tableau historique des progrès de Pesprit humain. Paris, Posthume.

D’Huy, J. (2013). A Phylogenetic Approach of Mythology and Its Archaeological Consequences. Rock Art Research, 30, 115-118.

Davidson, D. (1970). Mentals Events. In D. Davidson (Ed.), Essays on Actions and Events. New York: Oxford University Press.

De Nooy, W., Mrvar, A., \& Batagelj, V. (2011). Exploratory Social Network Analysis with Pajek (Structural Analysis in the Social Sciences). Cambridge: Cambridge University Press. https://doi.org/10.1017/CBO9780511996368

Dean, L. G., Kendal, R. L., Schapiro, S. J., Thierry, B., \& Laland, K. N. (2012). Identification of the Social and Cognitive Processes Underlying Human Cumulative Culture. Science, 335, 1114-1118. https://doi.org/10.1126/science.1213969

Deleuze, G., \& Guattari, F. (1991). Qu' est-ce que la philosophie? Lonrai: Les éditions de Minuit.

Diamond, J. (1997). Guns, Germs, and Steel: The Fates of Human Societies. New York: W.W. Norton and Company.

Fodor, J. A. (1983). The Modularity of Mind. Cambridge, MA: The MIT Press. https://doi.org/10.7551/mitpress/4737.001.0001

Fontana, W., \& Buss, L. W. (1994). What Would Be Conserved If "The Tape Were Played Twice"? Proceedings of the National Academy of Sciences of the United States of America, 91, 757-761. https://doi.org/10.1073/pnas.91.2.757

Gaucherel, C. (2012). Le quotidien du chercheur, une chasse aux fantômes? Montpellier: Eds Quae. https://doi.org/10.3917/quae.gauc.2013.01

Gaucherel, C. (2013). A Deep Unity between Scientific Disciplines. Open Journal of Philosophy, 3, 413-421. https://doi.org/10.4236/ojpp.2013.33061

Gaucherel, C. (2014). Ecosystem Complexity through the Lens of Logical Depth: Capturing Ecosystem Individuality. Biological Theory, 9, 440-451.

https://doi.org/10.1007/s13752-014-0162-2

Gaucherel, C., \& Jensen, H. J. (2012). Origins of Evolution: Non Acquired Characters Dominates over Acquired Characters in Changing Environment. Journal of Theoretical Biology, 304, 11-120. https://doi.org/10.1016/j.jtbi.2012.02.028 
Gaucherel, C., Boudon, F., Houet, T., Castets, M., \& Godin, C. (2012). Understanding Patchy Landscape Dynamics: Towards a Landscape Language. PLOS ONE, 7, e46064. https://doi.org/10.1371/journal.pone.0046064

Gaucherel, C., Théro, H., Puiseux, A., \& Bonhomme, V. (2017). Understand Ecosystem Regime Shifts by Modelling Ecosystem Development Using Boolean Networks. Ecological Complexity, 31, 104-114. https://doi.org/10.1016/j.ecocom.2017.06.001

Gell-Mann, M. (1994). The Quark and the Jaguar: Adventures in the Simple and the Complex. Manhattan, NY: St. Martin's Griffin. https://doi.org/10.1063/1.2808634

Gould, S. J. (1989). Wonderful Life: The Burgess Shale and the Nature of History. New York: W.W. Norton and Company.

Grimmett, G. R., \& Stirzaker, D. R. (1992). Probability and Random Processes. Oxford, UK: Clarendon Press.

Gruber, T. R. (1993). A Translation Approach to Portable Ontology Specifications. Knowledge Acquisition, 5, 199-220. https://doi.org/10.1006/knac.1993.1008

Haufe, C. (2015). Gould's Laws. Philosophy of Science, 82, 1-20. https://doi.org/10.1086/678979

Huneman, P. (2010). Topological Explanations and Robustness in Biological Sciences. Synthese, 177, 213-245. https://doi.org/10.1007/s11229-010-9842-Z

Jablonka, E., \& Lamb, M. J. (2005). Evolution in Four Dimensions. Cambridge, MA: The MIT Press.

Klavans, R., \& Boyack, K. (2009). Toward a Consensus Map of Science. Journal of the American Society for Information Science and Technology, 60, 455-476. https://doi.org/10.1002/asi.20991

Klein, E., \& Spiro, M. (1996). Le temps et sa flèche. Paris: Flammarion.

Maynard Smith, J., \& Szathmáry, E. (1995). The Major Transitions in Evolution. Oxford: Oxford University Press.

Mayr, E. (2004). What Makes Biology Unique? Cambridge, UK: Cambridge University Press. https://doi.org/10.1017/CBO9780511617188

Mitchell, S. D. (2000). Dimensions of Scientific Law. Philosophy of Science, 67, 242-265. https://doi.org/10.1086/392774

Morris, S. C. (2010). Evolution: Like Any Other Science It Is Predictable. Philosophical Transactions of the Royal Society B, 365, 133-145. https://doi.org/10.1098/rstb.2009.0154

Nagel, E. (1961). The structure of Science. London: Routledge and Kegan Paul.

Nelson, E. (2001). Warning Signs of a Possible Collapse of Contemporary Mathematics. In M. Heller \& W. H. Woodin (Eds.), Infinity: New Research Frontiers. Cambridge, UK: Cambridge University Press.

Oudiette, D., Dealberto, M. J., Uguccioni, G., Golmard, J. L., Merino-Andreu, M., Tafti, M., Garma, L., Schwartz, S., \& Arnulf, I. (2012). Dreaming without REM Sleep. Conscious and Cognition, 21, 1129-1140. https://doi.org/10.1016/j.concog.2012.04.010

Panza, M., \& Sereni, A. (2013). Plato's Problem: An Introduction to Mathematical Platonism. Hampshire, London: Palgrave Macmillan.

https://doi.org/10.1057/9781137298133

Pearce, T. (2012). Convergence and Parallelism in Evolution: A Neo-Gouldian Account. The British Journal for the Philosophy of Science, 63, 429-448.

https://doi.org/10.1093/bjps/axr046

Pesic, P. (2002). Seeing Double: Shared Identities in Physics, Philosophy, and Literature. 
Cambridge, MA: The MIT Press. https://doi.org/10.7551/mitpress/6138.001.0001

Popper, K. (1963). Conjectures and Refutations. Physics Today, 16, 80. https://doi.org/10.1063/1.3050617

Putnam, H. (1975). Mathematics, Matter, and Method: Philosophical Papers (Vol. 1). Cambridge, UK: Cambridge University Press.

Saitta, A. M., \& Saija, F. (2014). Miller Experiments in Atomistic Computer Simulations. Proceedings of the National Academy of Sciences of the United States of America, 111, 13768-13773. https://doi.org/10.1073/pnas.1402894111

Saunders, S., \& Wallace, D. (2008). Branching and Uncertainty. The British Journal for the Philosophy of Science, 59, 293-305. https://doi.org/10.1093/bjps/axn029

Sowa, J. F. (1995). Top-Level Ontological Categories. International Journal of Human Computer Studies, 43, 669-685. https://doi.org/10.1006/ijhc.1995.1068

Stengers, I. (1995). L'invention des sciences modernes. Paris: Flammarion.

Sterelny, K. (2015). Contingency and History. Philosophy of Science, 83, 521-539. https://doi.org/10.1086/687260

Tonelli, G. (1967). Kant and the Ancient Skeptics. Studien zu Kants philosophischer Entwicklung, hrsg.v. H. Heimsoeth, Hildesheim: Olms.

Van Fraassen, B. (1980). The Scientific Image. Oxford: Clarendon Press. https://doi.org/10.1093/0198244274.001.0001

Vermeij, G. J. (2006). Historical Contingency and the Purported Uniqueness of Evolutionary Innovations. Proceedings of the National Academy of Sciences of the United States of America, 103, 1804-1809. https://doi.org/10.1073/pnas.0508724103 